\title{
Social Networking for Pervasive Adaptation ${ }^{1}$
}

\author{
Stuart M. Allen, Marco Conti, Jon Crowcroft, Robin Dunbar, Pietro Lio', Jose Fernando Mendes \\ Refik Molva, Andrea Passarella, Ioannis Stavrakakis, Roger M. Whitaker ${ }^{2}$ \\ email: s.m.allen@cs.cardiff.ac.uk, marco.conti@iit.cnr.it, \\ jon.crowcroft@cl.cam.ac.uk, robin.dunbar@anthro.ox.ac.uk,p1219@cam.ac.uk, \\ jfmendes@fis.ua.pt, refik.molva@eurecom.fr, a.passarella@iit.cnr.it, \\ ioannis@di.uoa.gr,r.m.whitaker@cs.cardiff.ac.uk
}

\begin{abstract}
As technology progresses, we are seeing increasing numbers of small devices that have the capability to store, process and forward information in our everyday physical environments. Increasingly, this is opening up the opportunity to generate, collect and communicate new knowledge and information in diverse aspects of human life. However this raises significant challenges for communication and content acquisition, as new levels of flexibility, adaptation and dynamism are required. In particular, the traditional notion of always-on end-to-end network connectivity becomes inappropriate, and radical rethinking is necessary. The EU FET project Social Networking for Pervasive Adaptation (SOCIALNETS) seeks to overcome these challenges by understanding, modelling and exploiting the key characteristics that enable humans to adapt and exhibit agility beyond other species. Through developing the capacity for devices to socially network, we believe that there is significant potential to facilitate highly effective pervasive communication and content provision.
\end{abstract}

\section{Introduction}

As processors become smaller and cheaper and wireless personal and local area networking becomes ubiquitous, everyday environments are becoming increasing saturated with objects that have the potential to store, carry and forward new information. This is consistent with the vision of Mark Weiser [22] and more recently Adam Greenfield [6] whose well-recognised concepts of calm computing and everyware express the notion that this heavily distributed computing resource will seamlessly integrate and

\footnotetext{
${ }^{1}$ Authors are listed alphabetically.

${ }^{2}$ Corresponding author.
}

support human activities. This increase in distributed processors also extends to devices carried by the human, such as mobile phones, which increasingly offer the potential for users to generate their own content and communicate in new ways that have a low overhead.

This means that content is becoming increasingly unleashed into the physical world, where historically it has been confined to well-defined virtual spaces such as the Internet or Information systems. Consequently, this is generating new challenges for communication and acquisition of information, based on highly uncertain and transient interactions between devices, human mobility and lack of any centralization or common ownership. This generates the need for a radical rethink of existing engineering conventions with regard to networking. In wireless domains such as ad-hoc networking, the convention has been to emulate the wired world, generally seeking to sustain end-to-end connectivity irrespective of the application. The introduction of studies on delay-tolerance have sought to relax this, but temporary or transient connections are still viewed as an inconvenience to be overcome rather than as a natural phenonemon around which communication should be based.

Beyond communication, disconnectivity and highly uncertain interactions also have significant ramifications for content provision. Existing conventions such as pulling content from its source are impeded by uncertainty in connectivity and require new approaches to providing content that enable relevance to be maintained. This is particularly important as pervasive computing environments will further increase the long tail of content, where diversity of information will increase and relevance will relate to only a few. Cross cutting both communication and content provision are issues of trust and security. Decentralisation and lack of common ownership leave open a lack of incentive for cooperation and altruism between nodes.

Developing suitably adaptive methodologies to resolve 
these technical issues is a significant challenge, and piecewise or ad-hoc approaches to solving different aspects of the system are unlikely to be productive as they run the risk of being conceptually incompatible when combined. Consequently it is important that an overaching rationale is used to drive new development of different aspects, leading to a coherent new paradigm with widespread applicability.

Our observation motivating the SOCIALNETS project is the need for human-centricity. Communication and knowledge exchange are fundamental and natural tenets of human behaviour but current network architectures and approaches to content provision are artifically constructed and force natural human communication to follow engineering paradigms to facilitate transportation. The translation of such engineering conventions to wireless communication in the physical world has probably been one of the main roadblocks impeding the development of technologies such as mobile ad-hoc networks and sensor networks. In this paper we explain and justify how new approaches to humancentric adaptation are being developed from core characteristics of human behaviour and self-organisation.

\section{The SOCIALNETS Vision and Approach}

The SOCIALNETS project proposes that the solution to the challenges of pervasive networks lies in engaging the ways in which humans naturally structure interactions and relationships. These are constantly adapting to context and situation, and are persistently present in human society. By definition, they are intimately connected to the end-users of the technology. While there are an increasing number of natural metaphors used to provide self-organisation such as autonomic nervous systems and biological principles, human social networking spans diverse disciplines. These include a combination of ideas and concepts from social anthropology, complex systems, network engineering and computer science.

The overarching hypothesis of the SOCIALNETS project is that highly effective, evolve-able, trustworthy, adaptable and human centric pervasive communication systems will be realised by exploiting the core characteristics of human behaviour that lead to highly effective and $d y$ namic interpersonal relationships and social networks.

\subsection{The Nature of Human Relationships}

The basis for the SOCIALNETS approach social is the concept of the human relationship. This has inherent properties that are attractive as a basis for developing an adaptive communication paradigm. Namely:

- Human relationships are ties based on wide ranging issues, meaning that they can span diverse aspects such as trust, communication and content provision;
- Human relationships have casual and temporal dimensions, meaning that they are inherently transient and well suited to developing high levels of flexibility;

- Human relationships are the basis for performing complex functions, meaning that they can be engaged to facilitate more sophisticated functionality.

Taking this a stage further, multiple human relationships imply explicit and implicit social structures that have further desirable properties, such as:

- highly connected structures with distinct topological traits such as short paths;

- entirely decentralised formation and maintenance of social networks, with heterogeneity of participants;

- massively scalability and inclusivity with embedded hierarchies and protocols;

These provide a rich basis on which to harness new adaptivity for emerging pervasive and mobile computing environments.

\subsection{Manifesting Social Networks}

There are two ways in which the notions of social networks are relevant to pervasive adaptation. Firstly, physical social networks can be defined based on physical interactions between local devices. Alternatively, social networks can be defined in a virtual sense, where participants share some common interests or e-interactions.

Physical social networks give a basis by which the behaviour of humans can be captured by the pattern of interactions between devices in order to define and adapt a social structure based on familiarity, trust and other aspects. As the patterns of human behaviour change, the implied social relationships between devices will also change. As an example, in opportunistic forwarding, changes in relationships could trigger changes in the frequency with which copies of information are generated and/or forwarded from particular devices in order to maximise the probability of delivery of information to the intended recipients.

The virtual social network can be utilised to facilitate an adaptive data and knowledge base. Information can be replicated and distributed within a particular electronic social network so that relevance is maintained for community members. This is highly valuable because the technology rich environment is likely to contain a large number of artifacts of low relevance to many users. Consequently social networks give a basis for retaining and sharing appropriate knowledge without trying to obtain all artifacts necessarily from the information source.

Embedded in both the physical and virtual notions of electronic social networks are fundamental needs and new 
opportunities to provide security and trust. Security has particular relevance to preserving the integrity of physical interactions, while trust is of relevance to the acquisition and dissemination of appropriate content through cooperative relationships. Establishing trust and security between devices is a challenging issue. However, social network structures offer a basis to enhance trust and security provision by capitalising on communities of devices that have commonality between them, either physically or logically.

\subsection{Opportunistic Networks}

The flexible notion of relationships means that interactions in human social networks may occur at any point in time and independent of simultaneous linkage with others. Information may be retained and shared in addition to being forwarded. This is exactly consistent with the flexibility that is needed in pervasive communication scenarios. It is a highly desirable property for pervasive adaptation because it means that disconnections do not impede system operation. In this approach there is the potential to develop high levels of agility because dynamicity and uncertainty are inherent.

Although related to the current notions of opportunistic networking [16] and delay tolerance [12], where communication in the presence of incomplete paths is the underlying mission, the development of electronic social networks within this project significantly extends research results in these areas. Wireless interactions are instances of transient opportunistic networking between peers for data forwarding and knowledge sharing. These are closely integrated with human mobility and human interactions with each other and devices embedded in the physical environment and thus it is a natural extension to engage social network structures.

\subsection{The SOCIALNETS paradigm}

It is important to understand the paradigm we are seeking to develop is not intended as a replacement for the Internet, an existing communication system or any other networking paradigm. Instead, the social networking paradigm is intended to extend current communication functionality in a pervasive computing direction, so that future technology rich environments can be realised.

However, rather than incrementally improving functionality, the social networking paradigm represents a new and important generalization of all existing communication paradigms. This is because all existing communication systems operate to facilitate social interactions between individuals and also social interactions between individuals and technology. In the social networking paradigm we seek to develop via the SOCIALNETS project, the physical and electronic social interactions themselves are the basis for facilitating communication and knowledge provision throughout - there is no underlying network topology to be created, managed or evolved in facilitating communication. This is a radical proposition that removes the hypothesis that all communication must be based on networks with end-to-end connectivity.

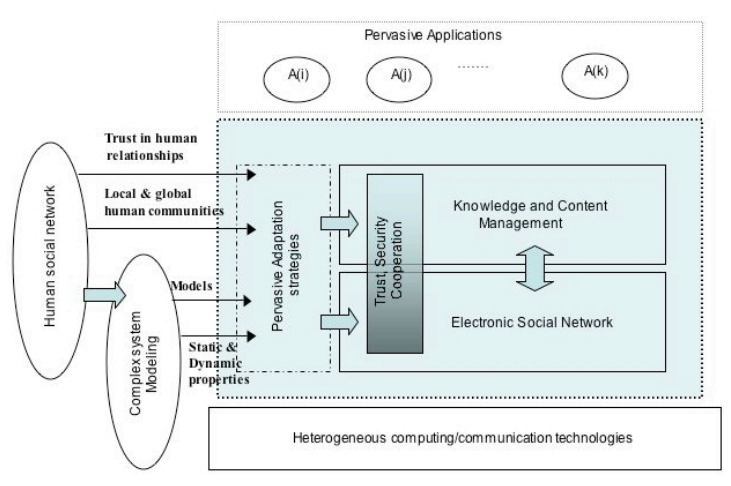

Figure 1. The SOCIALNETS paradigm

Figure 1 summarizes the SOCIALNETs approach to achieve pervasive adaptation in a world of ubiquitous, massive-scale, heterogeneous computing and communication devices. The pervasive adaptation paradigm is built by exploiting the properties and characteristics of human social networks. The analysis of these networks provides both direct and indirect contributions to the paradigm.

Firstly the characteristics and properties of human communities (e.g., local/global communities and trust in human relationships) constitute the qualitative basis for establishing dynamic trust relationships between humans and/or devices and for defining adaptive policies for information and communications built on the social context.

Secondly, the results of human social network studies are used for building complex-system models of the human social relationships. The analysis of these models then provide a quantitative characterisation of static and dynamic properties of human social networks on which pervasive adaptation paradigm is built upon.

The two proposed layers as shown in Figure 1 (electronic social network and knowledge and content management) will not be organized according to a strict layering approach, but rather cross-layering interactions will be introduced. As highlighted in the Figure 1, trust, security and cooperation policies require strict interactions among the two layers, but several other cross-feed channels are also envisaged.

\section{Basis for Development}

The background and motivation for the proposed paradigm is inherently interdisciplinary, with roots in so- 
cial anthropology, complex systems, network engineering and computer science. The starting point is understanding the properties of a social network.

\subsection{Characterisation of Social Networks}

In order to make use of human behavioural characteristics, we need to understand how social networks form themselves, maintain themselves and operate. Additionally, development of electronic social networks has added a further dimension by which people can "virtually" socialize and communicate. We contend that this must be considered from both a physical and social approach, involving physical dynamics, anthropological analysis and statistical modelling.

The work of Milgram [14] is recognised as a founding contribution concerning the understanding of the structure of human relations, conjecturing that only small sequences of relationships (typically five or six) are necessary in order to connect any pair of people. Retesting this in contemporary settings such as email and exploring the confidence and role of networkers has more recently taken place (e.g., [18]). The original experiment has also been used to explore the social connectivity in particular regions known for their closeness [17].

This idea of a small world has been reaffirmed in many settings, for example, Watts and Strogatz [21] demonstrate similar properties in various areas.

Beyond connectivity, contemporary research has recently sought to understand the dynamics and structure of social networks with respect to particular types of relationships. Broadly speaking, relationships can be classified by strength of mutual familiarity and intensity, which did not feature in Milgrams study. Strong relationships constitute personal relations where individuals who are directly known to each other and consequently give a basis for trust to be asserted firsthand.

Particular recent work suggests two important and relevant conclusions. Firstly, for a particular person (EGO) under consideration, there appears to be an upper limit on the typical number of individuals who can be known personally of about 150 (now commonly known as Dunbar's Number). Secondly, the members in an EGOs social network do not all have relationships of the same quality with EGO; rather, the network is structured into a series of circles of acquaintanceship which scale with a remarkably consistent ratio (almost exactly 3) [23].

Although these layers of acquaintanceship may in part be a function of the availability of time to interact with other individuals, there nonetheless seems to be a significant cognitive component: EGOs perceived relationship with another individual is a more or less linear function of that individuals location in the set of annuli, and this in turn correlates with the frequency with which that individual is contacted [10]. Thus, there appears to be important constraints on both the structure of natural social networks, on the frequency with individuals contact the members of their personal networks, and on the emotional ties that they can cope with.

\subsection{Physical Dynamics}

The physical dynamics of social relationships have been studied for a number of years [3]. Basic network notions, techniques, and concepts first elaborated in sociology, were later accepted and developed in graph theory and, more recently, in the statistical mechanics of complex networks. Key concepts have been developed and applied to capture dynamic behaviour and structural properties including transitivity (clustering), assortative and disassortative mixing (correlations between the numbers of connections of the nearest neighbours), k-cores, cliques, and communities, and spectral characteristics of networks.

Exponential and Markov models of social networks now are being studied by mathematicians, physicists, and computer scientists. These activities in statistical mechanics of complex networks has resulted in statistical physics and graph theory being applied to model and subsequently understand basic characteristics and behaviour in sociological networks. This research is revealing that some diverse realworld networks have common underlying physical properties relating to complexity and dynamics (e.g. [20, 15]).

In the case of networks of social contacts based on strong direct ties such as friendships, specific characteristics relate to very high density clustering (e.g., many triangles and loops) and assortative mixing. Consistent with anthropological evidence, these networks are usually small with strong finite size effects.

Beyond strong ties, the effects of weak ties in social networks are also crucial. The fundamental work of Granovetter [5] indicated the strength of weak ties. Examples of weak ties represent instances of acquaintances rather than friendship. Granovetters research argued that weak ties may provide a crucial role in forming bridges between social networks formed from strong ties in which there is a high degree of clustering and connectivity. Granovetters work identifies an interesting dichotomy: while weak ties are of a lower social quality (for example in terms of the trust that they can assert), they are crucial in facilitating information spread between the high density clusters that would otherwise be isolated. Consequently facilitating use of weak ties is crucial to dissemination.

However despite this, existing models of social networks still cannot give a satisfactory explanation and quantitative description of empirical observations of sociologists including Granovetters strength of weak ties phenomenon. This 
extends to quantitative understandings of even simple dynamic processes in social networks such as the spread of beliefs, gossip, rumour and perturbations. However we believe that the application of recent findings in the statistical mechanics of complex networks to social networks will make possible successful modelling of real networks and the processes taking place in them. This also extends to human mobility [4] where tracking the trajectory of mobile phone users has discovered inherent similarity in travel patterns could impact all phenomena driven by human mobility.

The project will be an important opportunity for discussing problems of data sharing for research projects dealing with complex systems, where use of proprietary and personal data may cause privacy violation. The role and space for modeling and simulation of social networks will open up the possibility of capturing the true differences between social groups and their use of technology.

\subsection{Electronic Communication in Social Networks}

Opportunistic and delay tolerant networks have emerged from relaxing the constraint on always-there end-to-end connectivity in a range of networks, from the internet to ad-hoc and sensor networks.

In the original specification of delay-tolerant network some estimated knowledge about future availability of links bridging regions of disconnection is assumed. The time during which such links are not available is accounted as a cost in traditional routing metrics, so that Internet-like route computation still remains feasible.

Opportunistic networking [16] further relaxes the concept of delay tolerance by assuming in general no apriori knowledge regarding the network topology, areas of disconnections or future availability of links. Therefore, route computation as in the traditional Internet algorithms (or even as in the MANET algorithms) is not feasible anymore. Instead, routes must be computed on-the-fly and hopby- hop while the message progresses towards the destination(s). Nodes carrying messages to be forwarded opportunistically evaluate if any other node they get in contact with could be a good next hop towards the destination, and hand the message over to it, if the case.

It has been demonstrated that opportunistic networks also provide a possible way to improve the capacity of multi-hop ad-hoc networks beyond the well-known theoretical limit found by Gupta and Kumar [8]. Additionally Grossglauser and Tse [7] have shown that an opportunistic network in which nodes act as carriers can achieve constant capacity, irrespective of the number of nodes in the network.

In the opportunistic networking field, there are a small number of emerging attempts to exploit social network fea- tures for structure and context $[11,1]$. This looks as a promising approach, as contacts between nodes is fundamentally tied with user behaviour, and, thus, with social network structures. However, as of today only limited aspects of social networks are exploited and their study has not been attempted in a systematic way with an underlying social networking paradigm on which to base technical development.

\subsection{Cooperation, Trust \& Security}

Trust and security are fundamental issues for the safe and effective operation of all communication systems. However, the increasing trend toward decentralization has resulted in significant challenges because traditional security solutions often require centralized online trusted authorities or certificate repositories, which are not well suited to securing ad-hoc or sensor networks and has led to emergence of enforcement techniques [13] for trust. This dichotomy becomes more significant for opportunistic networks where connectivity as well as centralization requirements are both relaxed.

Underlying these problems is the issue of cooperation. Behaving rationally, unrelated individual agents gain the most from uncooperative selfish behaviour (i.e., defection), independent of the behaviour of others. This may result in devices for example not reciprocating or passing data on for another. However, the resultant collective outcome is deficient or socially irrational. These problems are often called a "Tragedy of the Commons" [9], receiving attention in areas as diverse as economics, biology and socialanthropology. This widespread consideration arises because despite the individual rational choice being uncooperative behaviour, many organisations, species, societies and systems, exhibit cooperative behaviour, where individuals act altrustically for the socially rational outcome. This issue is highly relevant to social networks of devices because models to incentivize or enforce cooperation need to be developed.

It is considered to be a natural direction to pursue from the trust and security perspective because the notion of trust is fundamentally embedded in human interactions, behaviour and social networks [2]. Moreover, by bringing an interdisciplinary and socio-cognitive perspective, prospects for innovation are significantly increased.

\subsection{Data \& Knowledge Management}

Beyond transportation and delivery of data such as messages, opportunistic networking in technology richenvironments can be used to facilitate situated information provision. This aspect is important because the landscape will contain a vast range of information artifacts that can be em- 
bedded in the environment. However, individual artifacts are likely to be of interest and relevance to only a few participants.

Existing studies in this area have largely addressed content that is assumed static in the temporal domain. It is highly likely that in technology-rich environments, there will be a need to enable live interaction with the situated surrounding physical space in an electronic manner, taking account of the current scenario and resources within it.

Content, storage and forwarding also requires the need for co-operation. Developments in this area have looked at distributed selfish replication and caching through classical game-theoretic models and analysis as well as a microscopic approach to coping with mistreatment and selfishness. These approaches have ensured that mistreatment and exploitation of nodes is avoided. Thus cooperation is encouraged as it can only boost the gain of each and every party involved [19]. The scarcity of storage, the highly temporal/spatial content demand dynamics and the increased uncertainty of opportunistic networking create an environment that calls for innovative content storage and forwarding management schemes that exploit the structure and avoid the cavities of social networking.

\section{Conclusions}

The SOCIALNETS vision is to draw inspiration from the structure and characteristics of human social behaviour and interactions to derive a new paradigm for communication and information provision in massive scale, heterogeneous and trustworthy pervasive networks.

The successful realisation will require advances and collaboration between the fields of social anthropology, complex systems, network engineering and computer science.

Acknowledgement: This work has been supported by the SOCIALNETS project, grant agreement number 217141, funded by the EC seventh framework programme theme FP7-ICT-2007-8.2 for Pervasive Adaptation. See http://www.social-nets.eu/ for further details.

\section{References}

[1] C. Boldrini, M. Conti, and A. Passarella. Exploiting users' social relations to forward data in opportunistic networks: The hibop solution. Pervasive and Mobile Computing, 2008.

[2] G. Colombo, R. Whitaker, and S. Allen. Forming social networks of trust to incentivize cooperation. In Under review, 2008.

[3] S. N. Dorogovtsev and J. F. F. Mendes. Evolution of networks. Advances in Physics, 51:1079, 2002.

[4] M. Gonzalez, C. Hidalgo, and A.-L. Barabasi. Understanding individual human mobility patterns. Nature, 2008.
[5] M. S. Granovetter. The strength of weak ties. The American Journal of Sociology, 78(6):1360-1380, 1973.

[6] A. Greenfield. Everyware: The Dawning Age of Ubiquitous Computing. Peachpit Press, Berkeley, CA, USA, 2006.

[7] M. Grossglauser and D. N. C. Tse. Mobility increases the capacity of ad hoc wireless networks. IEEE/ACM Trans. Netw., 10(4):477-486, 2002.

[8] P. Gupta and P. Kumar. The capacity of wireless networks. Information Theory, IEEE Transactions on, 46(2):388-404, 2000.

[9] G. Hardin. The tragedy of the commons. Science, 162:1243$1248,1968$.

[10] R. A. Hill and R. I. M. Dunbar. Social network size in humans. Human Nature, 14:53-72, 2003.

[11] P. Hui, J. Crowcroft, and E. Yoneki. Bubble rap: socialbased forwarding in delay tolerant networks. In In Proceedings of the 9th ACM international Symposium on Mobile Ad Hoc Networking and Computing (MobiHoc '08)., 2008.

[12] K. Jain, J. Padhye, V. N. Padmanabhan, and L. Qiu. Impact of interference on multi-hop wireless network performance. In MobiCom '03: Proceedings of the 9th annual international conference on Mobile computing and networking, pages 66-80, New York, NY, USA, 2003. ACM Press.

[13] P. Michiardi and R. Molva. Core: A COllaborative REputation mechanism to enforce cooperation in mobile ad-hoc networks. In Proceedings of IFIP Communication and Multimedia Security conference, 2002.

[14] S. Milgram. The small world problem. Psychology Today, 2:60-67, 1967.

[15] M. E. J. Newman. Assortative mixing in networks. Physical Review Letters, 89:208701, 2002.

[16] L. Pelusi, A. Passarella, and M. Conti. Opportunistic networking: Data forwarding in disconnected mobile ad hoc networks. Communications Magazine, IEEE, 44(11):134141, 2006.

[17] A. Rowlands. Six degrees of welsh separation. http://news.bbc.co.uk/1/hi/wales/4141581.stm, 2005.

[18] D. Saxbe. Six degrees of separation: Two new studies test 'six degrees of separation' hypothesis. Psychology Today, 2003.

[19] G. Smaragdakis, N. Laoutaris, A. Bestavros, I. Matta, and I. Stavrakakis. Mistreatment-resilient distributed caching. Computer Networks, 51(11), 2007.

[20] S. H. Strogatz. Exploring complex networks. Nature, 410(6825):268-276, March 2001.

[21] D. J. Watts and S. H. Strogatz. Collective dynamics of "small-world" networks. Nature, 393:440-442, 1998.

[22] M. Weiser. Ubiquitous computing. Computer, 26(10):71$72,1993$.

[23] W. X. Zhou, D. Sornette, R. A. Hill, and R. I. M. Dunbar. Discrete hierarchical organization of social group sizes. PROCEEDINGS OF THE ROYAL SOCIETY B, 272:439, 2005. 